\title{
Impact of Sinus Rhythm Maintenance on Major Adverse Cardiac and Cerebrovascular Events after Catheter Ablation of Atrial Fibrillation: Insights from AF Frontier Ablation Registry
}

Keisuke Usuda ${ }^{1}$, Takeshi Kato ${ }^{1}$, Toyonobu Tsuda ${ }^{1}$, Hayato Tada ${ }^{1}$, Satoru Niwa ${ }^{1}$, Soichiro Usui $^{1}$, Kenji Sakata ${ }^{1}$, Kenshi Hayashi ${ }^{1}$, Hiroshi Furusho ${ }^{1}$, Masa-aki Kawashiri ${ }^{1}$, Masayuki Takamura $^{1}$, Takayuki Otsuka ${ }^{2}$, Akio Hirata ${ }^{3}$, Masato Murakami ${ }^{4}$, Mitsuru Takami ${ }^{5}$, Masaomi Kimura ${ }^{6}$, Hidehira Fukaya ${ }^{7}$, Shiro Nakahara ${ }^{8}$, Wataru Shimizu ${ }^{9}$, Tomoo Harada $^{10}$, Ken Okumura ${ }^{11}$, Teiichi Yamane ${ }^{12}$, Yukihiko Momiyama ${ }^{13}$, Kyoko Soejima ${ }^{14}$, Nobuhisa Hagiwara ${ }^{15}$, Masahide Harada ${ }^{16}$, Kazumasa Sonoda ${ }^{17}$, Masaru Inoue ${ }^{18}$, Koji Kumagai $^{19}$, Hidemori Hayashi ${ }^{20}$, Kazuhiro Satomi ${ }^{21}$, Yuji Watari ${ }^{22}$, Naoya Matsumoto ${ }^{23}$, Koichi Nagashima ${ }^{24}$, and Yasuo Okumura ${ }^{24}$

${ }^{1}$ Kanazawa University Graduate School of Medical Sciences

${ }^{2}$ The Cardiovascular Institute

${ }^{3}$ Osaka Police Hospital

${ }^{4}$ Shonan Kamakura General Hospital

${ }^{5}$ Kobe University Graduate School of Medicine

${ }^{6}$ Hirosaki University Graduate School of Medicine

${ }^{7}$ Kitasato University School of Medicine

${ }^{8}$ Dokkyo Medical University Koshigaya Hospital

${ }^{9}$ Nippon Medical School

${ }^{10}$ Saint Marianna University School of Medicine

${ }^{11}$ Saiseikai Kumamoto Hospital Cardiovascular Center

${ }^{12}$ Jikei University School of Medicine

${ }^{13}$ National Hospital Organisation Tokyo Medical Center

${ }^{14}$ Kyorin University School of Medicine

${ }^{15}$ Tokyo Women's Medical University

${ }^{16}$ Fujita Health University Hospital

${ }^{17}$ Tokyo Rinkai Hospital

${ }^{18}$ National Hospital Organization Kanazawa Medical Center

${ }^{19}$ Tohoku Medical and Pharmaceutical University Wakabayashi Hospital

${ }^{20}$ Juntendo University School of Medicine Graduate School of Medicine

${ }^{21}$ Tokyo Medical University

${ }^{22}$ Teikyo University Hospital

${ }^{23}$ Nihon University Hospital

${ }^{24}$ Nihon University School of Medicine Graduate School of Medicine

January 13, 2021 


\begin{abstract}
Introduction: The impact of catheter ablation for atrial fibrillation (AF) on cardiovascular events and mortality is controversial. We investigated the impact of sinus rhythm maintenance on major adverse cardiac and cerebrovascular events after AF ablation from a Japanese multicenter cohort of AF ablation. Methods and Results: We investigated 2737 consecutive patients (25.6\% female, mean age $63.4 \pm 10.3$ years) who underwent a first catheter ablation for AF from the Atrial Fibrillation registry to Follow the long-teRm Outcomes and use of aNTIcoagulants aftER Ablation (AF Frontier Ablation Registry). The primary endpoint was a composite of stroke, transient ischemic attack, cardiovascular events, and all-cause death. During a mean follow-up of 25.2 months, 2070 (75.6\%) patients were free from AF after catheter ablation, and the primary composite endpoint occurred in $122(4.5 \%)$ patients. The AF nonrecurrence group had a significantly lower incidence of the primary endpoint (1.7 per 100 person-years) compared with the AF recurrence group (3.2 per 100 person-years; $\mathrm{P}=0.001)$. The multivariate analysis revealed that freedom from AF (hazard ratio $0.57 ; 95 \%$ confidence interval $0.39-0.83 ; \mathrm{P}=0.003$ ) was independently associated with the incidence of the composite event. $\neg \neg$ Conclusion: In the multicenter cohort of AF ablation, sinus rhythm maintenance after catheter ablation was independently associated with lower rates of major adverse cardiac and cerebrovascular events.
\end{abstract}

Impact of Sinus Rhythm Maintenance on Major Adverse Cardiac and Cerebrovascular Events after Catheter Ablation of Atrial Fibrillation: Insights from AF Frontier Ablation Registry

Keisuke Usuda, ${ }^{1}$ MD; Takeshi Kato, ${ }^{1} \mathrm{MD}$; Toyonobu Tsuda,${ }^{1}$ MD; Hayato Tada, ${ }^{1}$ MD; Satoru Niwa,${ }^{1}$ MD; Soichiro Usui, ${ }^{1}$ MD; Kenji Sakata, ${ }^{1} \mathrm{MD}$; Kenshi Hayashi, ${ }^{1}$ MD; Hiroshi Furusho, ${ }^{1}$ MD; Masaaki Kawashiri, ${ }^{1} \mathrm{MD}$; Masayuki Takamura,${ }^{1} \mathrm{MD}$; Takayuki Otsuka, ${ }^{2} \mathrm{MD}$; Akio Hirata,${ }^{3} \mathrm{MD}$; Masato Murakami, ${ }^{4}$ MD; Mitsuru Takami, ${ }^{5}$ MD; Masaomi Kimura, ${ }^{6}$ MD; Hidehira Fukaya, ${ }^{7}$ MD; Shiro Nakahara,${ }^{8}$ MD; Wataru Shimizu,${ }^{9}$ MD; Tomoo Harada, ${ }^{10}$ MD; Ken Okumura, ${ }^{11} \mathrm{MD}$; Teiichi Yamane, ${ }^{12}$ MD; Yukihiko Momiyama, ${ }^{13}$ MD; Kyoko Soejima, ${ }^{14} \mathrm{MD}$; Nobuhisa Hagiwara, ${ }^{15} \mathrm{MD}$; Masahide Harada, ${ }^{16}$ MD; Kazumasa Sonoda, ${ }^{17}$ MD; Masaru Inoue ${ }^{18} \mathrm{MD}$; Koji Kumagai,${ }^{19} \mathrm{MD}$; Hidemori Hayashi, ${ }^{20} \mathrm{MD}$; Kazuhiro Satomi, ${ }^{21} \mathrm{MD}$; Yuji Watari, ${ }^{22} \mathrm{MD}$; Naoya Matsumoto,${ }^{23} \mathrm{MD}$; Koichi Nagashima,${ }^{24} \mathrm{MD}$; Yasuo Okumura, ${ }^{24} \mathrm{MD}$ on behalf of the AF Ablation Frontier Registry

From ${ }^{1}$ Department of Cardiovascular Medicine, Graduate School of Medical Science, Kanazawa University, Kanazawa, Japan; ${ }^{2}$ Department of Cardiology, The Cardiovascular Institute, Tokyo; ${ }^{3}$ Cardiovascular Division, Osaka Police Hospital, Osaka; ${ }^{4}$ Divison of Cardiology, Shonan-Kamakura General Hospital, Kanagawa; ${ }^{5}$ Saiseikai Nakatsu Hospital, Osaka; ${ }^{6}$ Divison of Cardiology, Pulmonary Medicine and Nephrology, Hirosaki University School of Medicine, Aomori; ${ }^{7}$ Kitasato University Hospital, Kanagawa; ${ }^{8}$ Dokkyo Medical University Saitama Medical Center, Saitama; ${ }^{9}$ Nippon Medical Hospital, Tokyo; ${ }^{10}$ St. Marianna University School of Medicine Hospital, Kanagawa; ${ }^{11}$ Saiseikai Kumamoto Hospital, Kumamoto; ${ }^{12}$ Tokyo Jikei University School of Medicine Hospital, Tokyo; ${ }^{13}$ National Hospital Organization Tokyo Medical Center, Tokyo; ${ }^{14}$ Kyorin University Hospital, Tokyo; ${ }^{15}$ Tokyo Women's Medical University Hospital, Tokyo; ${ }^{16}$ Fujita University Health Hospital, Aichi $;{ }^{17}$ Tokyo Rinkai Hospital, Tokyo; ${ }^{18}$ Ishikawa Prefectural Central Hospital, Ishikawa; ${ }^{19}$ Gunma Cardiovascular Center, Gunma $;{ }^{20}$ Juntendo University, Tokyo $;{ }^{21}$ Tokyo Medical University Hospital, Tokyo ${ }^{22}$ Teikyo University, Tokyo, ${ }^{23}$ Department of Cardiology, Nihon University Hospital Tokyo; and ${ }^{24}$ Division of Cardiology, Nihon University Itabashi Hospital, Tokyo

Short title: Impact of maintaining sinus rhythm by AF ablation

Acknowledgments: None

Data Availability: The deidentified participant data will not be shared.

Research Ethics: The protocol of this study was approved by Institutional Review Board of Nihon University Itabashi Hospital (RK-161213-6) along with the Institutional Review Boards of all participating institutions.

Funding sources: This study was supported by a research grant from Bristol-Meyers Squibb.

\title{
Disclosures:
}


The following authors have potential conflicts of interest: T.K. received a research grant from Daiichi-Sankyo, and lecture fees from Bristol-Myers Squibb, Daiichi-Sankyo and Nippon Boehringer Ingelheim, and honoraria for writing promotional material for Bristol-Myers Squibb. A.H. accepted remuneration from Nippon Boehringer Ingelheim, Bayer Healthcare, Bristol-Myers Squibb, Daiichi-Sankyo. M. Kimura accepted remuneration from Johnson \& Johnson K.K., Medtronic Japan, Bayer Healthcare. H. Fukaya received lecture fees from Nippon Boehringer Ingelheim and Daiichi-Sankyo. S. Nakahara received lecture fees from Bayer Healthcare, Daiichi-Sankyo, Bristol-Meyers Squibb, Pfizer Japan, and Nippon Boehringer Ingelheim. W.S. received research funding from Bristol-Myers Squibb, Daiichi-Sankyo, and Nippon Boehringer Ingelheim, and patent royalties/licensing fees from Daiichi-Sankyo, Pfizer Japan, Bristol-Myers Squibb, Bayer Healthcare, and Nippon Boehringer Ingelheim. T.H. serves as a consultant to Medtronic Japan and has received lecture fees from Daiichi-Sankyo. K.O. received remuneration from Nippon Boehringer Ingelheim, Daiichi-Sankyo, Johnson \& Johnson, and Medtronic Japan. K. Soejima received research funding from Daiichi-Sankyo and Nippon Boehringer Ingelheim, and accepted remuneration from Medtronic Japan, Jonson \& Jonson, and Abbott Medical Japan. N.H. accepted remuneration from Nippon Boehringer Ingelheim, Bristol-Myers Squibb, Bayer Healthcare, and research funding from Bayer Healthcare, Nippon Boehringer Ingelheim, Daiichi-Sankyo. M.H. received lecture fee from Nippon Boehringer Ingelheim, Daiichi-Sankyo, and Johnson \& Johnson. K. Satomi received research funding from BIOTRONIK Japan. N.M. received research funding from Daiichi-Sankyo. Y.O. received research funding from Bayer Healthcare, Daiichi-Sankyo, Bristol-Meyers Squibb, Nippon Boehringer Ingelheim, Pfizer Japan, TORAY, and Boston Scientific Japan and has accepted remuneration from Bayer Healthcare, Daiichi-Sankyo, and Bristol-Meyers Squibb. Other authors have no conflicts of interest.

Corresponding author: Takeshi Kato, MD

Department of Cardiovascular Medicine, Graduate School of Medical Science, Kanazawa University

13-1, Takara-machi, Kanazawa, Ishikawa 920-8641, Japan

Telephone: +81-76-265-2254

Fax: +81-76-234-4251

E-mail: takeshikato@me.com

Total word count: 4692 words including title page, abstract, text, references, and figures legends.

\section{ABSTRACT}

Introduction: The impact of catheter ablation for atrial fibrillation (AF) on cardiovascular events and mortality is controversial. We investigated the impact of sinus rhythm maintenance on major adverse cardiac and cerebrovascular events after AF ablation from a Japanese multicenter cohort of AF ablation.

Methods and Results: We investigated 2737 consecutive patients (25.6\% female, mean age $63.4 \pm 10.3$ years) who underwent a first catheter ablation for AF from the Atrial Fibrillation registry to Follow the longteRm Outcomes and use of aNTIcoagulants aftER Ablation (AF Frontier Ablation Registry). The primary endpoint was a composite of stroke, transient ischemic attack, cardiovascular events, and all-cause death. During a mean follow-up of 25.2 months, 2070 (75.6\%) patients were free from AF after catheter ablation, and the primary composite endpoint occurred in $122(4.5 \%)$ patients. The AF nonrecurrence group had a significantly lower incidence of the primary endpoint (1.7 per 100 person-years) compared with the AF recurrence group (3.2 per 100 person-years; $P=0.001)$. The multivariate analysis revealed that freedom from AF (hazard ratio $0.57 ; 95 \%$ confidence interval $0.39-0.83 ; P=0.003$ ) was independently associated with the incidence of the composite event.

Conclusion: In the multicenter cohort of AF ablation, sinus rhythm maintenance after catheter ablation was independently associated with lower rates of major adverse cardiac and cerebrovascular events.

Keywords: ablation; atrial fibrillation; stroke; cardiovascular disease; mortality.

INTRODUCTION 
Atrial fibrillation (AF) has attracted significant attention as a major risk factor for ischemic stroke, heart failure, and death. In fact, AF was independently associated with a 5-fold stroke risk, a 3-fold heart failure risk, and a 1.5-1.9-fold mortality risk based on large cohort studies. ${ }^{1-3}$ Although the elimination of AF by catheter ablation has been theoretically expected to reduce cardiovascular events and improve survival, the effect of sinus rhythm maintenance on the hard endpoints is not fully elucidated.

Considering the low incidence of major adverse cardiac and cerebrovascular events or death after catheter ablation of AF, a large population of AF ablation may be required to determine the effect of sinus rhythm maintenance on the hard endpoints. However, the identification of AF recurrence in large database-based registries is challenging. In fact, several large propensity-matched registry-based cohort studies ${ }^{4-8}$ have shown that catheter ablation of AF is significantly associated with a lower incidence of stroke, heart failure, and death compared with medical therapy alone. However, most of those studies lacked information on AF recurrence after catheter ablation, leaving unclear the impact of sinus rhythm maintenance on these adverse events. Although a few old retrospective studies have reported the impact of sinus rhythm maintenance after catheter ablation on clinical outcomes, the data from a large multicenter registry cohort in the era of direct oral anticoagulation (DOAC) is scarce.

To address this issue, we analyzed the data from the Atrial Fibrillation registry to Follow the long-teRm Outcomes and use of aNTIcoagulants aftER Ablation (AF Frontier Ablation Registry).

\section{METHODS}

\section{Data sources and participants}

The AF Frontier Ablation Registry (UMIN Clinical Trials Registry: UMIN000026849) is a multicenter cohort study whose study design has previously been described in detail. ${ }^{9}$ Briefly, the study included 3530 consecutive patients who underwent catheter ablation for AF at any of the 24 cardiovascular centers in Japan between August 2011 and July 2017. The clinical data, such as patient characteristics (e.g., age, sex, AF type, comorbidities, blood test results, echocardiographic characteristics, and drugs), catheter ablation findings, and follow-up data were retrieved from each hospital's medical records. The follow-up data included information as to whether the AF had relapsed after catheter ablation, whether oral anticoagulants (OACs) or antiarrhythmic drugs (AADs) had been discontinued, and whether adverse events such as ischemic stroke, hemorrhagic stroke, transient ischemic attack (TIA), heart failure, acute coronary syndrome, or all-cause death had occurred. The dates of AF recurrence and occurrence of these adverse events were also recorded.

In this analysis, we included patients from the AF Frontier Ablation Registry who underwent a first catheter ablation for AF and completed the follow-up more than 3 months after the procedure. To avoid the effects of multiple ablation sessions, we excluded patients who underwent catheter ablation of AF more than once.

The patients consented to the use of their anonymized clinical data for research purposes through the opt-out program. The study protocol was approved by the institutional review boards of all 24 hospitals where the patients were treated.

\section{Post-ablation follow-up}

In Japan, patients generally undergo routine outpatient follow-up every 1 to 3 months after catheter ablation for AF. During the follow-up periods, 12-lead electrocardiography, 24-h Holter monitoring, or cardiac event recorders are typically employed for detecting arrhythmias. The judgment of an AF recurrence was left to the discretion of the doctors of the respective institutions based on current guidelines, ${ }^{10-12}$ which describe any documented atrial tachyarrhythmia episode lasting more than $30 \mathrm{~s}$ is considered a recurrence. AF recurrence 3 months after catheter ablation was defined as late recurrence, and the isolated occurrence of AF during the 3-month blanking period was treated as nonrecurrence. The study patients were classified into AF recurrence group or AF nonrecurrence group according to the presence or absence of the late AF recurrence.

\section{Ablation protocol}


As previously described, ${ }^{13}, 14$ encircling pulmonary vein isolation was performed for patients registered in the AF Frontier Ablation Registry who required it through the use of a radiofrequency ablation catheter or cryoablation catheter, depending on the hospital's preference or type of catheter available at the time of the procedure. The ablation procedure was guided by a circular mapping catheter or a multiple-electrode catheter. The radiofrequency ablation employed an irrigated-tip contact force-sensing catheter or an irrigatedtip standard non-contact force-sensing catheter, and a 3-dimensional mapping system (EnSite NavX/Velocity [St. Jude Medical, St. Paul, MN, USA], CARTO [Biosense Webster, Irvine, CA, USA] or RHYTHMIA [Boston Scientific, Marlborough, MA, USA]). Cryoablation was performed with an Arctic Front Advance cardiac cryoablation catheter (Medtronic, Dublin, Ireland), and any touch-up ablation was performed with a standard irrigated-tip catheter. Some patients were injected intravenously with adenosine triphosphate after pulmonary vein isolation to expose dormant conduction between the pulmonary vein and left atrium. When acute pulmonary vein reconnection or dormant conduction occurred, touch-up ablation was performed. Additional linear ablation, such as tricuspid valve isthmus linear ablation, mitral isthmus linear ablation, and left atrial roof linear ablation, was performed at the physician's discretion. The residual potentials, including complex fractionated atrial electrograms in the left atrium, were ablated as appropriate.

\section{Study endpoints}

The primary endpoint of this study was a composite of major adverse cardiac and cerebrovascular events (defined as ischemic stroke, hemorrhagic stroke, TIA, cardiovascular events including hospitalization for heart failure, acute coronary syndrome, and other cardiovascular events and all-cause death). Such events occurring within 3 months after the procedure were considered procedure-related adverse events and were excluded from the endpoint.

\section{Statistics}

The continuous variables are listed as means \pm standard deviation, and the categorical variables are presented as numbers and percentages. Continuous variables were compared between the AF nonrecurrence group and the AF recurrence group using Student's t-test or the Wilcoxon Rank-Sum test. We employed the chi-squared test to compare the categorical variables between the groups. We tested for an association between the clinical variables and the primary composite endpoint using the multivariate Cox proportional hazards model with a stepwise procedure to evaluate the effect of covariates. We estimated the cumulative clinical adverse event rates using the Kaplan-Meier method and analyzed the differences with the log-rank test. Two-sided Pvalues $<0.05$ were considered statistically significant. We employed SPSS version 25.0.0.0 (IBM, Armonk, NY, USA) for all statistical analyses.

\section{RESULTS}

\section{Clinical characteristics}

After excluding 793 patients, we ultimately studied 2737 from the AF Frontier Ablation Registry who underwent first catheter ablation of AF and completed the follow-up more than 3 months after the procedure (Fig. 1). Table 1 shows the study population's clinical characteristics. The population's mean age was 63.4 \pm 10.3 years, and $25.6 \%$ were female. Patients with paroxysmal AF accounted for $62.7 \%$ of the population, and the mean $\mathrm{CHA}_{2} \mathrm{DS}_{2}-\mathrm{VASc}$ score was $2.1 \pm 1.5$. The mean left ventricular ejection fraction (LVEF) was $63.6 \% \pm 9.4 \%$, and the mean left atrial diameter (LAD) was $40.1 \pm 6.5 \mathrm{~mm}$. Warfarin was prescribed less frequently than direct oral anticoagulants (DOACs) (20.4\% vs. $79.6 \%$ ); $43 \%$ of the patients continued to take OACs throughout the follow-up period, and AADs were prescribed for $27.9 \%$ of the patients at the end of the follow-up.

\section{Late recurrence of atrial fibrillation}

Among the 2737 patients included in this analysis, 2070 (75.6\%) were free from AF, whereas $667(24.4 \%)$ experienced a late recurrence of AF. The follow-up for the AF nonrecurrence group and AF recurrence group was 4175 and 1573 person-years, respectively. Table 1 lists the patient characteristics of the AF nonrecurrence and AF recurrence groups. Compared with the AF recurrence group, the AF nonrecurrence group exhibited 
a significantly lower rate of persistent $\mathrm{AF}(34.7 \%$ vs. $45.3 \%, P<0.001)$, dyslipidemia $(34.9 \%$ vs. $39.3 \%, P=$ $0.039)$, nonischemic myocardiopathy ( $3.1 \%$ vs. $5.3 \%, P=0.01)$, warfarin use $(17.5 \%$ vs. $29.4 \%, P<0.001)$, continuation of OAC use ( $35.3 \%$ vs. $67.2 \%, P<0.001)$, AAD use $(21.3 \%$ vs. $27.9,<0.001)$, and smaller baseline $\operatorname{LAD}(39.7 \pm 6.4 \mathrm{~mm}$ vs. $41.5 \pm 6.4 \mathrm{~mm}, P<0.001)$.

\section{Clinical adverse events after catheter ablation}

Among the 2737 patients with AF included in this analysis, 122 (4.5\%) developed the primary composite endpoint, composed of major adverse cardiac and cerebrovascular events after ablation. The primary events included 18 ischemic strokes (14.8\%), 16 hemorrhagic strokes (13.1\%), 7 TIAs (5.7\%), 19 hospitalizations for heart failure (15.6\%), 19 acute coronary syndromes (15.6\%), 24 hospitalizations for other cardiovascular events (20\%), and 19 deaths from any cause (15.6\%). The overall incidence rate of the primary endpoint was 2.1 per 100 person-years.

The primary composite endpoint occurred in 72 patients of the AF nonrecurrence group (1.7 per 100 personyears) and 50 patients of the AF recurrence group (3.2 per 100 person-years). A Kaplan-Meier survival analysis indicated that the AF nonrecurrence group had a significantly lower cumulative incidence of the primary endpoint compared with the AF recurrence group $(P=0.001$ by the log-rank test) (Fig. 2).

\section{Factors associated with the primary composite endpoint}

Table 2 summarizes the association between the clinical variables and the primary composite endpoint. In the univariate Cox regression hazard model, older age, persistent AF, hypertension, diabetes, heart failure, old myocardial infarction, nonischemic myocardiopathy, higher $\mathrm{CHADS}_{2}$ score, higher $\mathrm{CHA}_{2} \mathrm{DS}_{2}$-VASc score, higher HAS-BLED score, lower LVEF, larger LAD, antiplatelet drug use, warfarin use compared with DOAC use, lower hemoglobin level, continuation of OAC use during the follow-up period, AAD use at the end of the follow-up, and late recurrence of AF were significantly associated with the occurrence of the primary composite endpoint.

The multivariate analysis revealed that older age (hazard ratio [HR] 1.05; 95\% confidence interval [CI] 1.03-1.07; $P<0.001$ ), hypertension (HR 1.58; 95\% CI 1.04-2.42; $P=0.034$ ), heart failure (HR 1.87; $95 \%$ CI 1.24-2.83; $P=0.003$ ), old myocardial infarction (HR 4.25; 95\% CI 2.44-7.41; $P<0.001$ ), nonischemic cardiomyopathy (HR 2.68; 95\% CI 1.54-4.67;P =0.001), and larger LAD (HR 1.18 per 5 -mm increase; $95 \%$ CI, 1.02-1.36; $P=0.027$ ) were independently associated with the incidence of the primary composite endpoint after catheter ablation. Conversely, freedom from AF recurrence was independently associated with a lower risk of the primary composite endpoint (HR 0.57; 95\% CI $0.39-0.83 ; P=0.003$ ).

We explored the association between freedom from AF recurrence and each component of the primary composite endpoint without adjusting for multiple testing. Freedom from AF was significantly associated with a lower risk of ischemic stroke/TIA (HR 0.43; 95\% CI 0.19-0.94; $P=0.035$ ) and hospitalization for heart failure (HR $0.21 ; 95 \%$ CI $0.083-0.54 ; P=0.001$ ).

\section{DISCUSSION}

\section{Main findings}

The main findings of this multicenter cohort study are as follows: (1) The incidence of the primary composite event of major adverse cardiac and cerebrovascular events after the first catheter ablation for AF was 2.1 per 100 person-years. (2) The primary composite endpoint occurred less frequently in the patients without late $\mathrm{AF}$ recurrence than in those with $\mathrm{AF}$ recurrence (incidence rates of 1.7 vs. 3.2 per 100 person-years, respectively). (3) In the multivariate analysis, older age (HR 1.05), hypertension (HR 1.58), heart failure (HR 1.87), old myocardial infarction (HR 4.25), nonischemic cardiomyopathy (HR 2.68), and larger LAD (HR 1.18) were independent factors associated with the primary composite endpoint, and freedom from AF recurrence was independently associated with a lower risk of the primary composite endpoint (HR 0.57).

\section{Association between atrial fibrillation and cardiac and cerebrovascular events}


Several previous epidemiological studies ${ }^{1-3}$ have identified $\mathrm{AF}$ as a strong risk factor for cardiac/cerebrovascular events and death. In AF, a loss of effective atrial contraction, atrial dilatation, endocardial denudation, abnormal changes in blood constituents, and inflammation occur in the atrium to fulfill Virchow's triad for thrombogenesis. ${ }^{15}$ Furthermore, loss of atrial contraction by AF reduces cardiac output by up to $25 \%$, particularly in diastolic dysfunction. ${ }^{16} \mathrm{AF}$ can also lead to arrhythmia-induced left ventricular dysfunction, which is induced by extracellular matrix remodeling, cellular remodeling, and defects in calcium ion handling. ${ }^{17}$ Based on these clinical and basic observations, maintenance of sinus rhythm can theoretically be expected to reduce the risk of cardiac and cerebrovascular events in patients with AF.

However, in the landmark the Atrial Fibrillation Follow-up Investigation of Rhythm Management (AFFIRM) trial, ${ }^{18}$ a rhythm-control therapy using antiarrhythmic drugs failed to show a survival advantage over a ratecontrol therapy in patients with AF and a high risk for stroke or death. On the other hand, the substudy of the AFFIRM trial found that the presence of sinus rhythm was independently associated with a survival benefit, whereas AAD use was associated with increased mortality. ${ }^{19}$ These results suggest that an effective method for maintaining sinus rhythm with fewer adverse effects, such as catheter ablation, might improve survival.

\section{Impact of sinus rhythm maintenance using catheter ablation on clinical hard endpoints}

A number of large propensity-matched registry-based cohort studies ${ }^{4-8}$ have shown that catheter ablation of AF is significantly associated with a lower incidence of stroke, heart failure, and death compared with medical therapy alone. However, most of those registry-based studies lacked information on AF recurrence after catheter ablation. The impact of sinus rhythm maintenance after catheter ablation on clinical outcomes has been investigated in only a few retrospective studies and our results were generally in accordance with these previous reports.

Lin et al. ${ }^{20}$ studied patients with $\mathrm{AF}$ whose $\mathrm{CHA}_{2} \mathrm{DS}_{2}$-VASc scores were [?]1 and who underwent catheter ablation in Taiwan. In that single-center study, freedom from AF after ablation was found to be a predictor of a lower incidence of major adverse cardiovascular events, including total vascular events (ischemic stroke, TIA, acute coronary artery events, and peripheral vascular events or pulmonary embolism) and death. Unfortunately, the number of study patients was somewhat small (118 in the nonrecurrence group and 56 in the recurrence group).

Hunter et al. ${ }^{21}$ tested 1273 patients with AF in a multicenter study in the UK and Australia and showed that freedom from $\mathrm{AF}$ after ablation was a predictor of stroke free survival. Ghanbari et al. ${ }^{22}$ studied 3058 patients with AF from a single-center registry in Michigan and found that sinus rhythm maintenance after catheter ablation of AF was independently associated with a lower risk of cardiac mortality, although the authors found no significant reduction in cerebrovascular events or mortality. These two studies are relatively old, and the recruited patients underwent catheter ablation before 2011, thereby predominantly using warfarin instead of DOACs. To identify the effect of sinus rhythm maintenance more clearly, patients taking DOACs might be more suitable than those taking warfarin given that DOACs are associated with fewer adverse events. ${ }^{23-26}$ All of the patients in our study underwent AF ablation after 2011, and DOACs were predominantly prescribed in $78.4 \%$ of the patients. Our study is the first to demonstrate the favorable effect of sinus rhythm maintenance after AF ablation on hard endpoints from a large multicenter cohort in the DOAC era. Interestingly, the reduction in the primary endpoint by sinus rhythm maintenance per se was considerable (HR 0.57) and was comparable to that of the AFFIRM study (HR 0.54). ${ }^{18}, 19$

\section{Predictors for major adverse events after catheter ablation of AF}

In this study, older age, hypertension, heart failure, old myocardial infarction, nonischemic cardiomyopathy, and larger LAD were independent factors associated with the major adverse cardiac and cerebrovascular events in AF patients after catheter ablation. These predictors were independent of AF recurrence and generally concordant with those identified in the previous studies for AF patients treated without catheter ablation. ${ }^{27-30}$ 


\section{Limitations}

The current study had several limitations, the first of which is that it is not free from the intrinsic limitations of a retrospective analysis. There were significant differences in the baseline characteristics between the AF nonrecurrence and AF recurrence groups, although the results were adjusted for such covariates. A causal relationship between the lower incidence of the primary endpoint and sinus rhythm maintenance by catheter ablation is therefore not clear. Second, although the AF recurrence rate of this study was comparable to that of previous reports, ${ }^{21,} 22$ we cannot deny that asymptomatic AF recurrence might have been overlooked.

\section{CONCLUSION}

In the multicenter cohort of AF ablation, sinus rhythm maintenance after catheter ablation was independently associated with lower rates of major adverse cardiac and cerebrovascular events.

\section{References}

1. Wolf PA, Abbott RD, Kannel WB. Atrial fibrillation as an independent risk factor for stroke: the Framingham Study. Stroke 1991; 22: 983-988, doi:10.1161/01.str.22.8.983.

2. Stewart S, Hart CL, Hole DJ, McMurray JJ. A population-based study of the long-term risks associated with atrial fibrillation: 20-year follow-up of the Renfrew/Paisley study. Am J Med 2002; 113: 359-364, doi:10.1016/s0002-9343(02)01236-6.

3. Benjamin EJ, Wolf PA, D'Agostino RB, Silbershatz H, Kannel WB, Levy D. Impact of atrial fibrillation on the risk of death: the Framingham Heart Study. Circulation 1998; 98: 946-952, doi:10.1161/01.cir.98.10.946.

4. Bunch TJ, Crandall BG, Weiss JP, May HT, Bair TL, Osborn JS, et al. Patients treated with catheter ablation for atrial fibrillation have long-term rates of death, stroke, and dementia similar to patients without atrial fibrillation. J Cardiovasc Electrophysiol 2011; 22: 839-845, doi:10.1111/j.1540-8167.2011.02035.x.

5. Chang CH, Lin JW, Chiu FC, Caffrey JL, Wu LC, Lai MS. Effect of radiofrequency catheter ablation for atrial fibrillation on morbidity and mortality: a nationwide cohort study and propensity score analysis. Circ Arrhythm Electrophysiol 2014; 7: 76-82, doi:10.1161/circep.113.000597.

6. Friberg L, Tabrizi F, Englund A. Catheter ablation for atrial fibrillation is associated with lower incidence of stroke and death: data from Swedish health registries. Eur Heart J 2016; 37: 2478-2487, doi:10.1093/eurheartj/ehw087.

7. Modin D, Claggett B, Gislason G, Hansen ML, Worck R, Johannessen A, et al. Catheter ablation for atrial fibrillation is associated with lower incidence of heart failure and death. Europace 2020; 22: 74-83, doi:10.1093/europace/euz264.

8. Reynolds MR, Gunnarsson CL, Hunter TD, Ladapo JA, March JL, Zhang M, et al. Health outcomes with catheter ablation or antiarrhythmic drug therapy in atrial fibrillation: results of a propensity-matched analysis. Circ Cardiovasc Qual Outcomes 2012; 5: 171-181, doi:10.1161/circoutcomes.111.963108.

9. Okumura Y, Nagashima K, Arai M, Watanabe R, Yokoyama K, Matsumoto N, et al. Current Status and Clinical Outcomes of Oral Anticoagulant Discontinuation After Ablation for Atrial Fibrillation in Japan Findings From the AF Frontier Ablation Registry. Circ J 2019; 83: 2418-2427, doi:10.1253/circj.CJ-19-0602.

10. January CT, Wann LS, Alpert JS, Calkins H, Cigarroa JE, Cleveland JC, Jr., et al. 2014 AHA/ACC/HRS guideline for the management of patients with atrial fibrillation: a report of the American College of Cardiology/American Heart Association Task Force on Practice Guidelines and the Heart Rhythm Society. J Am Coll Cardiol 2014; 64: e1-76, doi:10.1016/j.jacc.2014.03.022.

11. Calkins H, Hindricks G, Cappato R, Kim YH, Saad EB, Aguinaga L, et al. 2017 HRS/EHRA/ECAS/APHRS/SOLAECE expert consensus statement on catheter and surgical ablation of atrial fibrillation. Europace 2018; 20: e1-e160, doi:10.1093/europace/eux274. 
12. Kirchhof P, Benussi S, Kotecha D, Ahlsson A, Atar D, Casadei B, et al. 2016 ESC Guidelines for the management of atrial fibrillation developed in collaboration with EACTS. Eur Heart J 2016; 37: 2893-2962, doi:10.1093/eurheartj/ehw210.

13. Haïssaguerre M, Jaïs P, Shah DC, Takahashi A, Hocini M, Quiniou G, et al. Spontaneous initiation of atrial fibrillation by ectopic beats originating in the pulmonary veins. N Engl J Med 1998; 339: 659-666, doi:10.1056/nejm199809033391003.

14. Kuck KH, Brugada J, Fürnkranz A, Metzner A, Ouyang F, Chun KR, et al. Cryoballoon or Radiofrequency Ablation for Paroxysmal Atrial Fibrillation. N Engl J Med 2016; 374: 2235-2245, doi:10.1056/NEJMoa1602014.

15. Watson T, Shantsila E, Lip GY. Mechanisms of thrombogenesis in atrial fibrillation: Virchow's triad revisited. Lancet 2009; 373: 155-166, doi:10.1016/s0140-6736(09)60040-4.

16. Naito M, David D, Michelson EL, Schaffenburg M, Dreifus LS. The hemodynamic consequences of cardiac arrhythmias: evaluation of the relative roles of abnormal atrioventricular sequencing, irregularity of ventricular rhythm and atrial fibrillation in a canine model. Am Heart J 1983; 106: 284-291, doi:10.1016/00028703(83)90194-1.

17. Gopinathannair R, Etheridge SP, Marchlinski FE, Spinale FG, Lakkireddy D, Olshansky B. ArrhythmiaInduced Cardiomyopathies: Mechanisms, Recognition, and Management. J Am Coll Cardiol 2015; 66: 17141728, doi:10.1016/j.jacc.2015.08.038.

18. Wyse DG, Waldo AL, DiMarco JP, Domanski MJ, Rosenberg Y, Schron EB, et al. A comparison of rate control and rhythm control in patients with atrial fibrillation. N Engl J Med 2002; 347: 1825-1833, doi:10.1056/NEJMoa021328.

19. Corley SD, Epstein AE, DiMarco JP, Domanski MJ, Geller N, Greene HL, et al. Relationships between sinus rhythm, treatment, and survival in the Atrial Fibrillation Follow-Up Investigation of Rhythm Management (AFFIRM) Study. Circulation 2004; 109: 1509-1513, doi:10.1161/01.Cir.0000121736.16643.11.

20. Lin YJ, Chao TF, Tsao HM, Chang SL, Lo LW, Chiang CE, et al. Successful catheter ablation reduces the risk of cardiovascular events in atrial fibrillation patients with CHA2DS2-VASc risk score of 1 and higher. Europace 2013; 15: 676-684, doi:10.1093/europace/eus336.

21. Hunter RJ, McCready J, Diab I, Page SP, Finlay M, Richmond L, et al. Maintenance of sinus rhythm with an ablation strategy in patients with atrial fibrillation is associated with a lower risk of stroke and death. Heart 2012; 98: 48-53, doi:10.1136/heartjnl-2011-300720.

22. Ghanbari H, Başer K, Jongnarangsin K, Chugh A, Nallamothu BK, Gillespie BW, et al. Mortality and cerebrovascular events after radiofrequency catheter ablation of atrial fibrillation. Heart Rhythm 2014; 11: 1503-1511, doi:10.1016/j.hrthm.2014.05.003.

23. Connolly SJ, Ezekowitz MD, Yusuf S, Eikelboom J, Oldgren J, Parekh A, et al. Dabigatran versus warfarin in patients with atrial fibrillation. N Engl J Med 2009; 361: 1139-1151, doi:10.1056/NEJMoa0905561.

24. Granger CB, Alexander JH, McMurray JJ, Lopes RD, Hylek EM, Hanna M, et al. Apixaban versus warfarin in patients with atrial fibrillation. N Engl J Med 2011; 365: 981-992, doi:10.1056/NEJMoa1107039.

25. Yamashita T, Koretsune Y, Yang Y, Chen SA, Chung N, Shimada YJ, et al. Edoxaban vs. Warfarin in East Asian Patients With Atrial Fibrillation - An ENGAGE AF-TIMI 48 Subanalysis. Circ J 2016; 80: 860-869, doi:10.1253/circj.CJ-15-1082.

26. Chai-Adisaksopha C, Hillis C, Isayama T, Lim W, Iorio A, Crowther M. Mortality outcomes in patients receiving direct oral anticoagulants: a systematic review and meta-analysis of randomized controlled trials. J Thromb Haemost 2015; 13: 2012-2020, doi:10.1111/jth.13139. 
27. Abhayaratna WP, Seward JB, Appleton CP, Douglas PS, Oh JK, Tajik AJ, et al. Left atrial size: physiologic determinants and clinical applications. J Am Coll Cardiol 2006; 47: 2357-2363, doi:10.1016/j.jacc.2006.02.048.

28. Gage BF, Waterman AD, Shannon W, Boechler M, Rich MW, Radford MJ. Validation of clinical classification schemes for predicting stroke: results from the National Registry of Atrial Fibrillation. JAMA 2001; 285: 2864-2870, doi:10.1001/jama.285.22.2864.

29. Risk factors for stroke and efficacy of antithrombotic therapy in atrial fibrillation. Analysis of pooled data from five randomized controlled trials. Arch Intern Med 1994; 154: 1449-1457.

30. Yamamoto K, Ikeda U, Furuhashi K, Irokawa M, Nakayama T, Shimada K. The coagulation system is activated in idiopathic cardiomyopathy. J Am Coll Cardiol 1995; 25: 1634-1640, doi:10.1016/07351097(95)00049-a.

Figure legends

Figure 1. Flowchart of the study selection process. Abbreviation: AF, atrial fibrillation.

Table 1. Clinical characteristics of the study patients. Abbreviations: AF, atrial fibrillation; TIA, transient ischemic attack; $\mathrm{Cr}$, creatinine; $\mathrm{Hb}$, hemoglobin; OAC, oral anticoagulant; $\mathrm{AAD}$, antiarrhythmic drugs.

Figure 2. Kaplan-Meier curve for the primary endpoint in patients without AF recurrence and those with AF recurrence. Abbreviation: AF, atrial fibrillation.

Table 2. Cox regression analysis for variables of the primary endpoint. Abbreviations: AF, atrial fibrillation; TIA, transient ischemic attack; $\mathrm{Cr}$, creatinine; Hb, hemoglobin; DOAC, direct oral anticoagulant; OAC, oral anticoagulant; AAD, antiarrhythmic drug; HR, hazard ratio; CI, confidence interval.

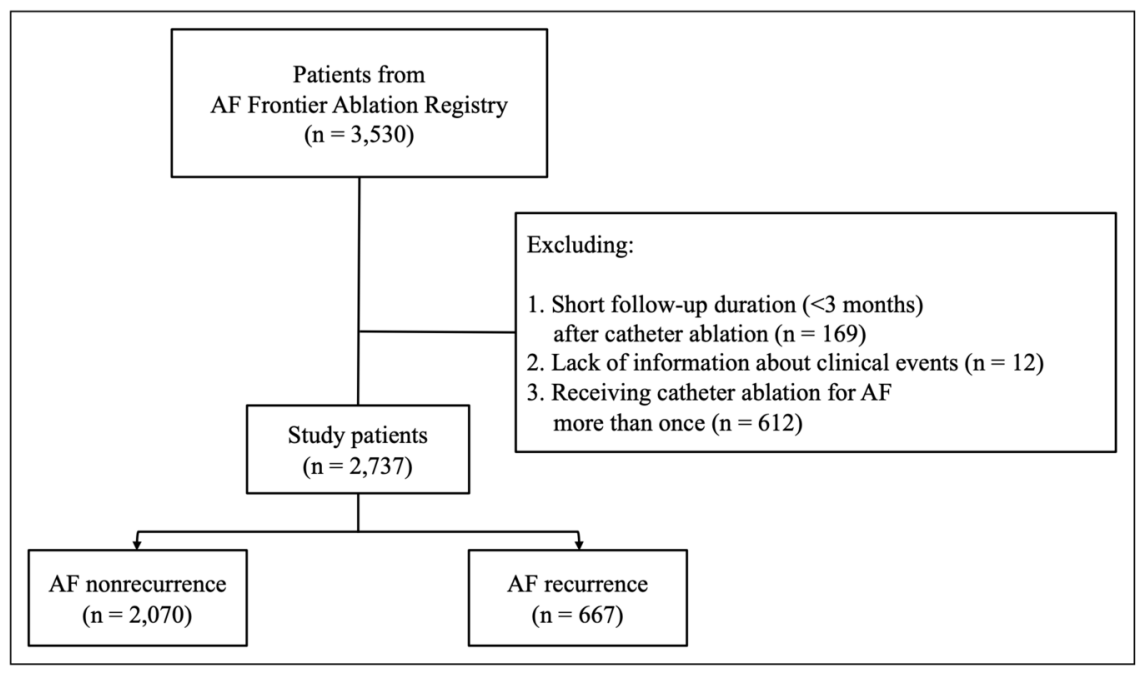

Figure 1. 
Table 1.

\begin{tabular}{|c|c|c|c|c|}
\hline & Total $(\mathbf{n}=\mathbf{2 , 7 3 7 )}$ & AF nonrecurrence group $(n=2,070)$ & AF recurrence group $(\mathrm{n}=667)$ & P value \\
\hline Age (years) & $63.4 \pm 10.3$ & $63.3 \pm 10.3$ & $63.7 \pm 10.3$ & 0.42 \\
\hline Female gender & $700(25.6 \%)$ & $522(25.2 \%)$ & $178(26.7 \%)$ & 0.45 \\
\hline Body mass index $\left(\mathrm{kg} / \mathrm{m}^{2}\right)$ & $24.0 \pm 3.7$ & $23.9 \pm 3.7$ & $24.2 \pm 3.6$ & 0.13 \\
\hline \multicolumn{5}{|l|}{ Type of AF } \\
\hline Paroxysmal & $1716(62.7 \%)$ & $1351(65.3 \%)$ & $365(54.7 \%)$ & $<0.001$ \\
\hline Persistent & $1021(37.3 \%)$ & $719(34.7 \%)$ & $302(45.3 \%)$ & \\
\hline \multicolumn{5}{|l|}{ Comorbidities } \\
\hline Hypertension & $1506(55.0 \%)$ & $1125(54.3 \%)$ & 381 (57.1\%) & 0.21 \\
\hline Diabetes & $453(16.6 \%)$ & $341(16.5 \%)$ & $112(16.8 \%)$ & 0.85 \\
\hline Dyslipidemia & $984(36.0 \%)$ & $722(34.9 \%)$ & $262(39.3 \%)$ & 0.039 \\
\hline History of stroke/TIA & $228(8.3 \%)$ & $163(7.9 \%)$ & $65(9.7 \%)$ & 0.13 \\
\hline Heart failure & $478(17.5 \%)$ & $363(17.5 \%)$ & $115(17.2 \%)$ & 0.86 \\
\hline Old myocardial infarction & $62(2.3 \%)$ & $44(2.1 \%)$ & $18(2.7 \%)$ & 0.39 \\
\hline Nonischemic cardiomyopathy & $99(3.6 \%)$ & $64(3.1 \%)$ & $35(5.3 \%)$ & 0.01 \\
\hline CHADS $_{2}$ score & $1.2 \pm 1.1$ & $1.2 \pm 1.1$ & $1.2 \pm 1.1$ & 0.16 \\
\hline $\mathrm{CHA}_{2} \mathrm{DS}_{2}$-VASc score & $2.1 \pm 1.5$ & $2.0 \pm 1.5$ & $2.1 \pm 1.6$ & 0.087 \\
\hline HAS-BLED score & $0.8 \pm 0.8$ & $0.7 \pm 0.8$ & $0.8 \pm 0.8$ & 0.16 \\
\hline \multicolumn{5}{|l|}{ Echocardiographic variables } \\
\hline Left ventricular ejection fraction (\%) & $63.6 \pm 9.4$ & $63.7 \pm 9.4$ & $63.2 \pm 9.7$ & 0.26 \\
\hline Left atrial diameter $(\mathrm{mm})$ & $40.1 \pm 6.5$ & $39.7 \pm 6.4$ & $41.5 \pm 6.4$ & $<0.001$ \\
\hline Antiplatelet drugs use & $201(7.3 \%)$ & $149(7.3 \%)$ & $52(7.8 \%)$ & 0.61 \\
\hline Warfarin use & $558(20.4 \%)$ & $362(17.5 \%)$ & $196(29.4 \%)$ & $<0.001$ \\
\hline \multicolumn{5}{|l|}{ Blood test } \\
\hline $\mathrm{Cr}(\mathrm{mg} / \mathrm{dL})$ & $0.94 \pm 0.79$ & $0.92 \pm 0.74$ & $0.98 \pm 0.90$ & 0.13 \\
\hline $\mathrm{Hb}(\mathrm{g} / \mathrm{dL})$ & $14.2 \pm 1.5$ & $14.2 \pm 1.5$ & $14.2 \pm 1.6$ & 0.59 \\
\hline $\begin{array}{l}\text { Continuation of OACs } \\
\text { (During follow-up period) }\end{array}$ & $1178(43.0 \%)$ & $730(35.3 \%)$ & $448(67.2 \%)$ & $<0.001$ \\
\hline $\begin{array}{l}\text { AAD use } \\
\text { (At the end of follow up) }\end{array}$ & $762(27.9 \%)$ & $440(21.3 \%)$ & $762(27.9 \%)$ & $<0.001$ \\
\hline Follow-up period (months) & $25.2 \pm 16.2$ & $24.2 \pm 15.4$ & $28.3 \pm 18.0$ & $<0.001$ \\
\hline Developping the composite event & $122(4.5 \%)$ & $72(3.5 \%)$ & $50(7.5 \%)$ & $<0.001$ \\
\hline
\end{tabular}

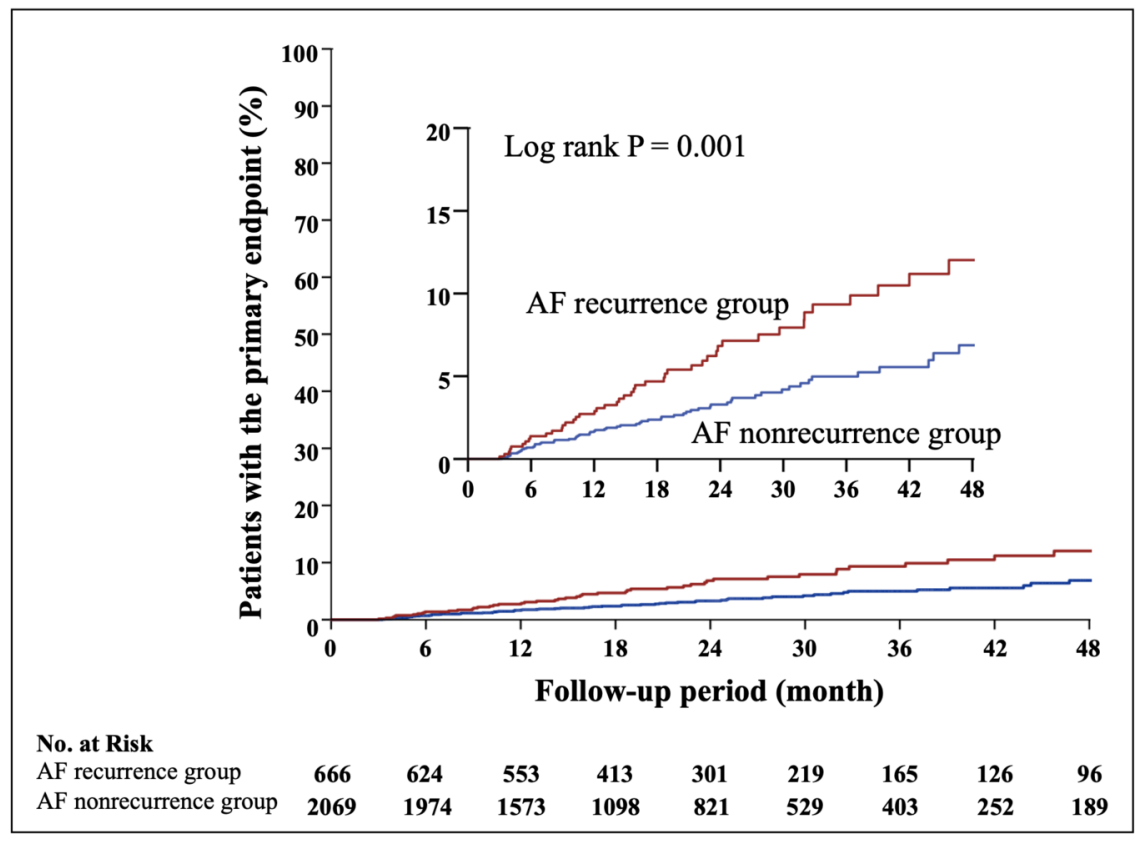

Figure 2. 
Table 2.

\begin{tabular}{|c|c|c|c|c|c|c|}
\hline & \multicolumn{3}{|c|}{ Univariate analvsis } & \multicolumn{3}{|c|}{ Multivariate analvsis } \\
\hline & HR & $95 \% \mathrm{CI}$ & $P$ value & HR & $95 \% \mathrm{CI}$ & $P$ value \\
\hline Age $(+1$ year $)$ & 1.06 & $1.03-1.08$ & $<0.001$ & 1.05 & $1.03-1.07$ & $<0.001$ \\
\hline Female & 0.79 & $0.52-1.21$ & 0.28 & & & \\
\hline Body mass index $\left(+1 \mathrm{~kg} / \mathrm{m}^{2}\right)$ & 0.98 & $0.93-1.03$ & 0.48 & & & \\
\hline Persistent AF & 1.56 & $1.10-2.23$ & 0.014 & & & \\
\hline \multicolumn{7}{|l|}{ Comorbidities } \\
\hline Hypertension & 1.94 & $1.30-2.88$ & 0.001 & 1.58 & $1.04-2.42$ & 0.034 \\
\hline Diabetes & 1.81 & $1.21-2.70$ & 0.004 & & & \\
\hline Dyslipidemia & 1.36 & $0.95-1.95$ & 0.089 & & & \\
\hline History of stroke/TIA & 1.4 & $0.80-2.44$ & 0.24 & & & \\
\hline Heart failure & 2.68 & $1.84-3.90$ & $<0.001$ & 1.87 & $1.24-2.83$ & 0.003 \\
\hline Old myocardial infarction & 6.06 & 3.53-10.4 & $<0.001$ & 4.25 & $2.44-7.41$ & $<0.001$ \\
\hline Nonischemic cardiomyopathy & 3.59 & $2.12-6.08$ & $<0.001$ & 2.68 & $1.54-4.67$ & 0.001 \\
\hline CHADS $_{2}$ score & 1.53 & $1.34-1.75$ & $<0.001$ & - & - & - \\
\hline $\mathrm{CHA}_{2} \mathrm{DS}_{2}$-VASc score & 1.46 & $1.32-1.62$ & $<0.001$ & - & - & - \\
\hline HAS-BLED score & 1.58 & $1.30-1.91$ & $<0.001$ & - & - & - \\
\hline \multicolumn{7}{|l|}{ Echocardiographic variables } \\
\hline Left ventricular ejection fraction $(+1 \%)$ & 0.97 & $0.96-0.99$ & $<0.001$ & & & \\
\hline Left atrial diameter $(+5 \mathrm{~mm})$ & 1.39 & $1.22-1.59$ & $<0.001$ & 1.18 & $1.02-1.36$ & 0.027 \\
\hline Antiplatelet drug use & 3.1 & $2.03-4.73$ & $<0.001$ & & & \\
\hline Warfarin use (compared with DOAC use) & 1.96 & $1.35-2.86$ & $<0.001$ & & & \\
\hline \multicolumn{7}{|l|}{ Blood test } \\
\hline $\mathrm{Cr}(+1 \mathrm{mg} / \mathrm{dL})$ & 1.11 & $0.99-1.27$ & 0.078 & & & \\
\hline $\mathrm{Hb}(+1 \mathrm{~g} / \mathrm{dL})$ & 0.79 & $0.71-0.89$ & $<0.001$ & & & \\
\hline Freedom from $\mathrm{AF}$ recurrence & 0.54 & 0.380 .78 & 0.001 & 0.57 & $0.39-0.83$ & 0.003 \\
\hline $\begin{array}{l}\text { Continuation of OAC } \\
\text { (During follow-up period) }\end{array}$ & 1.64 & $1.15-2.34$ & 0.007 & & & \\
\hline $\begin{array}{l}\text { AAD use } \\
\text { (At the end of follow-up) }\end{array}$ & 1.57 & $1.08-2.28$ & 0.017 & & & \\
\hline
\end{tabular}

